\title{
Colour vision of diabetics
}

\author{
F D GREEN,' I M GHAFOUR,' D ALlAN, ' T BARRIE,' E McCluRE,' \\ AND W S FOULDS'
}
From the 'Tennent Institute of Ophthalmology, University of Glasgow, Western Infirmary, Glasgow, G11 6NT, and the ${ }^{2}$ West of Scotland Health Boards, Department of Clinical Physics and Bio-Engineering, 11 West
Graham Street, Glasgow G4 9LF

\begin{abstract}
Summary The Farnsworth-Munsell 100-hue test has been assessed as a screening test for the detection of diabetic retinopathy likely to benefit from laser photocoagulation therapy. Two hundred and thirty-two diabetic eyes of 126 patients were tested. The results were assessed both for total error score relative to age and for the presence of polarity. Although the incidence of abnormal colour discrimination was found to correlate with the severity of retinopathy, the test was not sufficiently selective to be of value as a screening test in the detection of retinopathy requiring treatment.
\end{abstract}

Diabetes is a common cause of blindness in the UK. The survey by Sorsby' showed it to be the fifth commonest cause of blindness in England and Wales, while in a recent study ${ }^{2}$ confined to the west of Scotland diabetes ranked as the fourth commonest cause.

Treatment by photocoagulation is now widely available for patients with the more serious ocular complications of the disease, particularly exudative maculopathy and proliferative retinopathy. Some $1 \%$ of the population have diagnosed diabetes, while about $10 \%$ of diabetics develop retinopathy which threatens sight. ${ }^{3}$ Such retinopathy, particularly of the proliferative variety, may be asymptomatic for a considerable time, yet early detection and timely laser photocoagulation can prevent progression to blindness. ${ }^{4}$

An effective screening test for the early detection of those diabetics with sight-threatening retinopathy would be of considerable value. Lakowski, Aspinall, and Kinnear ${ }^{5}$ found that diabetic patients whose colour vision was assessed with the FarnsworthMunsell 100-hue test ${ }^{6}$ had higher error scores than did normal observers of similar ages. If retinopathy was present, the error score was further increased.

We have assessed the Farnsworth-Munsell 100-hue test in relation to its ability to separate diabetics with sight-threatening retinopathy from those with milder ocular disease.

Correspondence to Professor W S Foulds.

\section{Materials and methods}

The Farnsworth-Munsell 100-hue test was performed by patients attending a diabetic medical clinic for routine ocular screening. The fundi of these patients were graded into one of four groups after ophthalmoscopic examination by an ophthalmologist. In addition a group of normal people, drawn from the staff of the department, were tested. No one with a known condition likely to impair colour discrimination was included in this group.

The groups examined were as follows: Group $\mathrm{N}-$ Normal persons. Group NR-Diabetics who showed no diabetic retinopathy on ophthalmoscopy. Group $B$-Background retinopathy. Those with opthalmoscopically visible retinal haemorrhages, hard exudates and microaneurysms with retention of good vision; patients with soft exudates (cotton wool spots) were excluded. Group $P$-Proliferative retinopathy; those with abnormal new vessels in the retina. Group E-Exudative maculopathy; those with macular or paramacular hard exudates and/or macular oedema and visual acuity below 6/18.

The test was performed under Illuminant $C$ lighting conditions at an illumination level of approximately 200 lux in a VeriVide lighting cabinet (Leslie Hubble Ltd), and no limit was placed on the time taken. The test was performed monocularly and any appropriate near correction was used by the subject. The colour caps were presented to the observers in four sets of 21 or 22 caps as described by Farnsworth. ${ }^{6}$ 
Table 1 Numbers of 100-hue test results analysed by two methods of assessment for various categories of diabetic retinopathy. Both assessments include two subjects, one with one eye in group $N R$ and the other in group $B$, and the second with one eye in group $B$ and one in group $E$. The totals take account of these cases

\begin{tabular}{|c|c|c|c|c|}
\hline & \multicolumn{2}{|c|}{$\begin{array}{l}\text { Total error score } \\
\text { assessment }\end{array}$} & \multicolumn{2}{|c|}{$\begin{array}{l}\text { Polarity } \\
\text { assessment }\end{array}$} \\
\hline & Eyes & Subjects & Eyes & Subjects \\
\hline Group N, normals & 18 & 16 & 18 & 16 \\
\hline Group NR, no retinopathy & 115 & 59 & 115 & 59 \\
\hline Group B, background retinopathy & 55 & 31 & 28 & 15 \\
\hline Group $\mathrm{P}$, proliferative retinopathy & 42 & 24 & 13 & 8 \\
\hline Group E, exudative maculopathy & 20 & 12 & 5 & 3 \\
\hline All diabetics & 232 & 124 & 161 & 83 \\
\hline All subjects & 250 & 140 & 179 & 99 \\
\hline
\end{tabular}

In most cases the scoring was calculated by an automatic electronic system (OE 8500A F-M Hue Tester, Osprey Electronics) and in the remainder by the testing optician. With both methods the error for each colour cap was calculated and plotted according to the method of Farnsworth, ${ }^{6}$ and the total error score for all 85 caps was computed. Two hundred and fifty eyes in 140 subjects were tested. The numbers tested in the various groups are shown in Table 1.

Total error scores were judged abnormal if they fell outside the 95th percentile for age as published by Verriest et al. ${ }^{7}$ for monocular testing without previous binocular experience, and normal otherwise.

The 100-hue test score charts were also analysed to determine whether any showed a polarity of error score, that is to say whether there were two regions of higher error scores lying roughly diametrically opposite on the circular score chart. In an attempt to remove subjectivity from the identification of any such cases, a mathematical method based on the use of Fourier series was developed and applied to the test data. Full details of the mathematical method are given elsewhere. ${ }^{8}$

In essence, from the 85 individual cap error scores on the chart computer calculations generate a figure which is a measure of how marked is any polarity and indicate the axis of confusion. The detection threshold for polar distributions was similar for the computer method and for an experienced 100-hue test assessor. The test results for 179 eyes in 99 subjects were analysed for the presence of a polarity by the computer-aided method (Table 1 ).

\section{Results}

Abnormaly high errors were found for a significant number of diabetic patients whatever the grade of retinopathy present. No significant differences were found between the proportions of males and females
Table 2 Numbers of abnormally high 100-hue test total error scores in normal subjects and in patients with various grades of diabetic retinopathy

\begin{tabular}{lrr}
\hline & \multicolumn{1}{c}{ Eyes } & \multicolumn{1}{c}{ Subjects $^{*}$} \\
\hline Group N, normals & $3(17 \%)$ & $3(19 \%)$ \\
Group NR, no retinopathy & $28(24 \%)$ & $19(32 \%)$ \\
Group B, background retinopathy & $18(33 \%)$ & $11(35 \%)$ \\
Group P, proliferative retinopathy & $21(50 \%)$ & $14(58 \%)$ \\
Group E, exudative maculopathy & $19(95 \%)$ & $12(100 \%)$ \\
\hline
\end{tabular}

*Abnormal score for one or both eyes.

with abnormal error scores in the various retinopathy classes, and accordingly the results presented here are not separated by sex.

The results are summarised in Table 2, where it will be seen that the proportion of abnormally high scores rose from $17 \%$ in normal eyes to $95 \%$ in the case of eyes with exudative maculopathy. $32 \%$ of diabetic patients without retinopathy showed an abnormal test result in one or both eyes, while $58 \%$ of those with proliferative retinopathy did so. From these results it is clear that many diabetics have abnormal colour discrimination and that colour vision generally deteriorates as the retinopathy becomes more severe. The results are shown graphically in Fig. 1.

The total error scores are further analysed in Table 3 , where the effectiveness of using an abnormally high total error score as an indicator of the presence of serious retinopathy-either proliferative retinopathy or exudative maculopathy - is explored. From the table it can be seen that when used in this way the sensitivity of the test (the proportion of serious retinopathies detected) was $65 \%$ (40 out of 62 eyes). The specificity (the proportion of non-serious retinopathy showing normal colour vision) was $73 \%$. If the data are examined on the basis of patients, the sensitivity and specificity become $72 \%$ and $66 \%$ respectively.

It was found that only 10 of the 179100 -hue test charts examined showed a detectable polarity. The 10 positive findings were composed of six eyes from five subjects with no retinopathy and four eyes of four subjects from the proliferative group. The evidence is insufficient to say whether the incidence of polarity

Table 3 Analysis of total error score data for serious diabetic retinopathy (groups $P$ and $E$ combined) and nonserious retinopathy (groups $N R$ and $B$ combined). Figures are for numbers of eyes.

\begin{tabular}{llcc}
\hline & Serious & Non-serious & Totals \\
\hline Abnormal score & 40 & 46 & 86 \\
Normal score & 22 & 124 & 146 \\
Totals & 62 & 170 & 232 \\
\hline
\end{tabular}




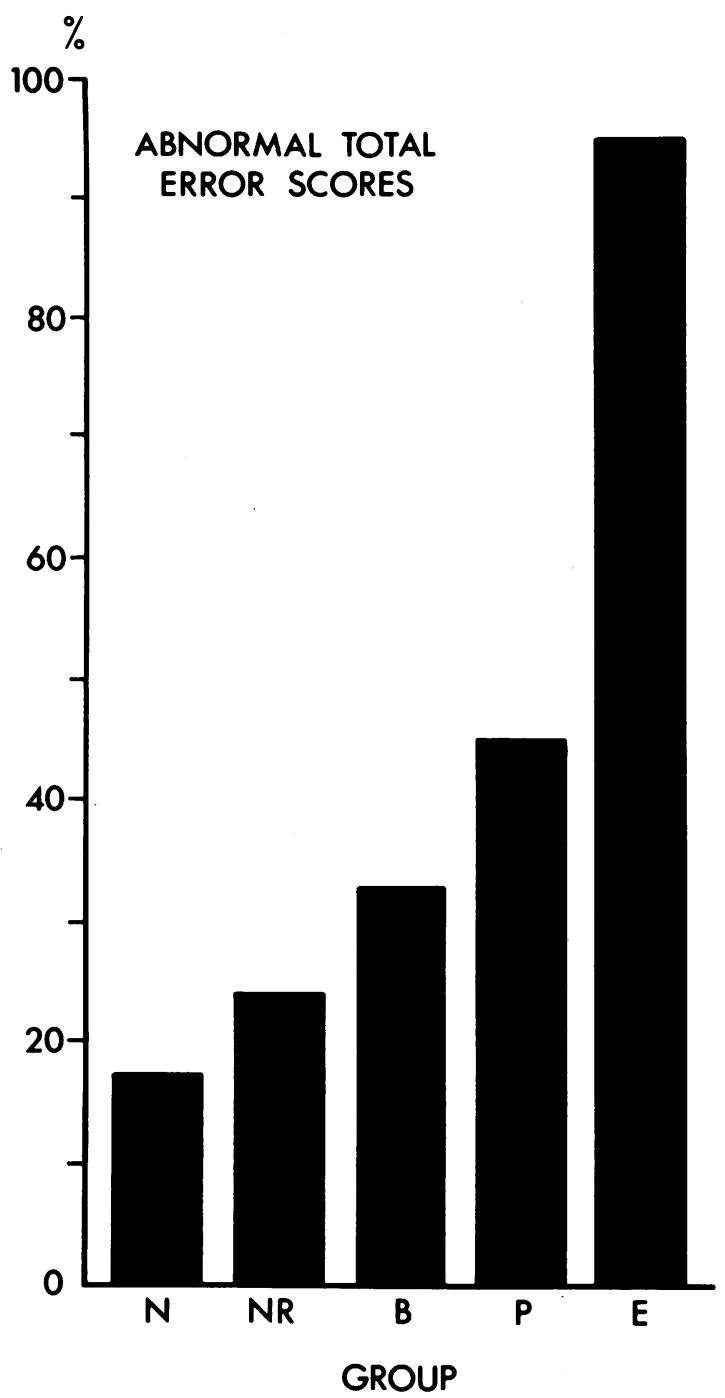

Fig. 1 Percentages of eyes returning abnormally high total error scores for the 100-hue test. Subject categories: $N$-normals, NR-no retinopathy, B-background retinopathy, $P$-proliferative retinopathy, $E$-exudative maculopathy.

varied in any systematic way with the grade of retinopathy.

Where a polarity was detected there was no ambiguity in the axis of colour confusion. For eight eyes of eight subjects, four each from the no retinopathy and proliferative groups, the axis of confusion ran between caps 81 and 7 and was therefore a blueyellow axis, while the remaining two charts, for the two eyes of a 74-year-old man with no retinopathy, showed red-green axes running through caps 27 and 29.

\section{Discussion}

It is well established that colour vision is affected by diabetic retinopathy. Colour vision is a function of the cones, and patients with exudative maculopathy would be expected to demonstrate the most marked defects of colour vision. Our results confirm this; $95 \%$ of eyes $(100 \%$ of subjects) with exudative maculopathy had an abnormally high total error score for their age.

In some cases of proliferative retinopathy areas of capillary closure may be situated peripherally in the retina where they might be expected not to influence colour vision, while in other patients intraretinal microvascular abnormalities or capillary closure may affect the macular circulation and lead to a deterioration of colour vision. In our proliferative retinopathy group $50 \%$ of eyes ( $58 \%$ of subjects) had significantly defective colour vision.

In addition to these more or less expected observations we also found significant defects of colour vision in many diabetics with no clinically visible retinopathy or only simple background retinopathy. In these two categories $24 \%$ and $32 \%$ of eyes $(32 \%$ and $35 \%$ of subjects) respectively showed abnormally high error scores. The mechanism of this deterioration of colour discrimination remains obscure, but it may result from early damage to the cones or their neuronal connections, which cannot be detected ophthalmoscopically, as previously suggested by Kinnear."

Our primary aim was to determine whether the 100 -hue test might be of value in separating those patients with retinopathy requiring treatment from those who did not. It is clear from the results presented in Table 3 that the test does not provide an effective means of doing this. About $35 \%$ of those with serious retinopathy are not detected by the criterion of the total error score; this figure is too high for the test to be considered an effective screening method.

A study of the contrast sensitivity performance of diabetics produced a rather similar result. ${ }^{10}$ Although diabetics as a group displayed a significant reduction in contrast sensitivity as measured by the Arden grating test, the changes were not sufficiently large in relation to the normal range for effective identification of individual diabetics with retinopathy on the evidence of the test result alone.

Of the 161 diabetic eyes examined for polarity only 10 had a significant polarity. The red-green polarity found in the results of a 74-year-old man may well have been a congenital colour vision defect, leaving only eight positive results to be attributed to diabetic retinopathy. Colour vision testing with the PickfordNicholson anomaloscope ${ }^{5}$ has shown that diabetics 
have an increased incidence of poor blue-yellow discrimination. It may be that our investigations have not shown this to any extent because, as suggested by Taylor," the divisions between the four boxes of colour caps in the 100-hue test fall at disadvantageous points of the colour sequence for the demonstration of these particular defects.

\section{CONCLUSIONS}

The Farnsworth-Munsell 100-hue test demonstrates deteriorating colour discrimination with increasing severity of diabetic retinopathy. The test is not on its own sufficiently specific to be of value as a screening test for the identification of serious diabetic retinopathy requiring laser treatment.

This work was supported by the Scottish Home and Health Department (grant No. K/MRC/50/C186). Mrs Jennifer Murray typed the manuscript.

\section{References}

1 Sorsby $\mathrm{S}$. The incidence and causes of blindness in England and Wales 1963-1968. Rep Publ Health Med Sub (Lond) 1972; 128.
2 Ghafour IM, Allan D, Foulds WS. Common causes of blindness and visual handicap in the west of Scotland. Br J Ophthalmol 1983; 67: 209-13.

3 Scobie IN, MacCuish AC, Barrie T, Green FD, Foulds WS. Serious retinopathy in a diabetic clinic: prevalence and therapeutic implications. Lancet 1981; ii: 520-1.

4 Cheng H. Photocoagulation and diabetic retinopathy. Br Med J 1979 ; i: $365-6$.

5 Lakowski R, Aspinall PA, Kinnear PR. Association between colour vision losses and diabetes mellitus. Ophthalmic Res 1972-3; 4: 145-59.

6 Farnsworth D. The Farnsworth-Munsell 100-hue and dichotomous tests for colour vision. J Opt Soc Am 1943; 33: 568-72.

7 Verriest G, Van Laethem J, Uvijls A. A new assessment of the normal ranges of the Farnsworth-Munsell 100-hue test scores. Am J Ophthalmol 1982; 93: 635-42.

8 Allan D. Fourier analysis and the Farnsworth-Munsell 100-hue test. Ophthalmic Physiol Opt in press.

9 Kinnear PR. The colour discrimination of diabetics. University of Edinburgh, 1965: MSc Thesis.

10 Ghafour IM, Foulds WS, Allan D, McClure E. Contrast sensitivity in diabetic subjects with and without retinopathy. $\mathrm{BrJ}$ Ophthalmol 1982; 66: 492-5.

11 Taylor WOG. Problems in performance and interpretation of Farnsworth's 100-hue test. In: Verriest G, ed. Mod Probl Ophthalmol 1974; 13: 73-8. 